\title{
Glomerulocystic Disease
}

National Cancer Institute

\section{Source}

National Cancer Institute. Glomerulocystic Disease. NCI Thesaurus. Code C123015.

A condition characterized by dilatation of the Bowman space and affecting more than $5 \%$

of the glomeruli. 